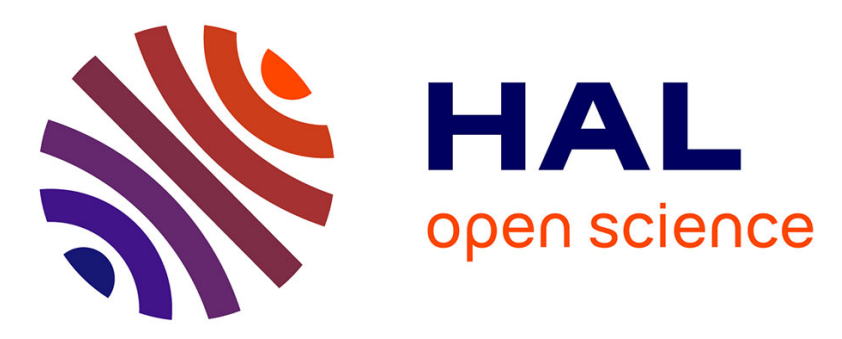

\title{
Effect of the angle of attack of a rectangular wing on the heat transfer enhancement in channel flow at low Reynolds number
}

Assadour Khanjian, Charbel Habchi, Serge Russeil, Daniel Bougeard, Thierry Lemenand

\section{To cite this version:}

Assadour Khanjian, Charbel Habchi, Serge Russeil, Daniel Bougeard, Thierry Lemenand. Effect of the angle of attack of a rectangular wing on the heat transfer enhancement in channel flow at low Reynolds number. Heat and Mass Transfer, 2017, 54 (5), pp.1441-1452. 10.1007/s00231-017-2244-8 . hal-02527875

\section{HAL Id: hal-02527875 \\ https://univ-angers.hal.science/hal-02527875}

Submitted on 1 Apr 2020

HAL is a multi-disciplinary open access archive for the deposit and dissemination of scientific research documents, whether they are published or not. The documents may come from teaching and research institutions in France or abroad, or from public or private research centers.
L'archive ouverte pluridisciplinaire HAL, est destinée au dépôt et à la diffusion de documents scientifiques de niveau recherche, publiés ou non, émanant des établissements d'enseignement et de recherche français ou étrangers, des laboratoires publics ou privés. 


\title{
Effect of the angle of attack of a rectangular wing on the heat transfer enhancement in channel flow at low Reynolds number
}

\author{
Assadour Khanjian $^{1,2} \cdot$ Charbel Habchi $^{3} \cdot$ Serge Russeil $^{1} \cdot$ Daniel Bougeard $^{1} \cdot$ Thierry Lemenand $^{4}$
}

Received: 10 May 2017 / Accepted: 24 November 2017

(C) Springer-Verlag GmbH Germany, part of Springer Nature 2017

\begin{abstract}
Convective heat transfer enhancement can be achieved by generating secondary flow structures that are added to the main flow to intensify the fluid exchange between hot and cold regions. One method involves the use of vortex generators to produce streamwise and transverse vortices superimposed to the main flow. This study presents numerical computation results of laminar convection heat transfer in a rectangular channel whose bottom wall is equipped with one row of rectangular wing vortex generators. The governing equations are solved using finite volume method by considering steady state, laminar regime and incompressible flow. Three-dimensional numerical simulations are performed to study the effect of the angle of attack $\alpha$ of the wing on heat transfer and pressure drop. Different values are taken into consideration within the range $0^{\circ}<\alpha<30^{\circ}$. For all of these geometrical configurations the Reynolds number is maintained to $R e=456$. To assess the effect of the angle of attack on the heat transfer enhancement, Nusselt number and the friction factor are studied on both local and global perspectives. Also, the location of the generated vortices within the channel is studied, as well as their effect on the heat transfer enhancement throughout the channel for all $\alpha$ values. Based on both local and global analysis, our results show that the angle of attack $\alpha$ has a direct impact on the heat transfer enhancement. By increasing its value, it leads to better enhancement until an optimal value is reached, beyond which the thermal performances decrease.
\end{abstract}

\section{Introduction}

Heat transfer enhancement is essential for many engineering applications such as heat exchangers [1,2], electronic equipments [3], high temperature gas turbines [4] or nuclear power plants [5]. Among all heat transfer enhancement existing methods, some are based on the idea of generating a secondary flow which is added to the main flow to intensify the fluid exchange between hot and cold regions in the system. One of these methods involves the use of vortex generators (VG) which can be either fixed on the wall or punched on it $[6,7]$.

Assadour Khanjian

assadour.khanjian@liu.edu.lb

1 IMT Lille Douai, Industrial Energy Department, F59500 Douai, France

2 Mechanical Engineering Department, Lebanese International University LIU, Beirut, Lebanon

3 Mechanical Engineering Department, Notre Dame University Louaize NDU, Zouk Mikael, Lebanon

4 ISTIA, LARIS, Université d'Angers, EA 7315 Angers, France
The main idea of the vortex generation (VG) enhancement method is to create streamwise vortices and improve heat transfer knowing that the presence of the VG leads to a relatively minor increase in the pressure losses [6, 8-10]. There are different techniques of vortex-induced heat transfer enhancement. Based on their function, these techniques can be divided into three categories: active VG, passive VG and compound VG [11] which involves the use of both active and passive techniques. On the one hand, active vortex generation needs external power like electric or acoustic fields, mechanical devices or surface vibration. On the other hand, passive VG do not need external power but use special aerodynamic surface geometry to create longitudinal or roll vortices.

Four basic configurations of vortex generators are widely discussed in the open literature: delta wings (DW), rectangular wings (RW), delta-winglet pair (DWP) and rectangular winglet pair (RWP). When the trailing edge is attached to the plate, the VG is a wing, when the chord length is attached to the plate, it is called a winglet [6]. Because of the great interest found in the domain of vortex generators, different shapes of VG are studied and may also be found in recent papers: trapezoidal VG used in the high efficiency vortex (HEV) static mixer [12, 13], Zhou and Feng [14] studied the heat transfer 
enhancement due to linear and curved VG having punched holes on them, Wang et al. [15] investigated the semi dimple VG.

There are two major types of vortices generated by VG: transverse vortices (TV) and longitudinal vortices (LV). Transverse vortices are mainly recirculation regions in the near wake of the VG and affect unfavorably the heat transfer locally in that region. These vortices were found to induce local overheated regions which may be critical in case of exothermal chemical reactions for example [13]. Meanwhile, longitudinal vortices positively act upon heat transfer through three mechanisms: developing boundary layers, vortices and flow destabilization [16].. New boundary layers are generated around the surface of the VG, vortices are generated as a result of the VG and flow is less stable due to the reversed flows. Longitudinal vortices are 3D swirling motion creating upwash and downwash flow phenomena that persists over a long distance downstream the VG in the main flow direction [16].

Fiebig et al. [17] studied the effect of different VG configurations on heat transfer and pressure losses. The emphases of their study were the drag coefficient, the aspect ratios and the influence of the angle of attack for Reynolds number values in the range [2000-8000]. They have found out that the drag created by the VG is independent of the Reynolds number and the geometry of the VG, but is proportional to the VG frontal area.

Several experimental and numerical investigations were previously conducted to study the heat transfer enhancement downstream VG. Wu and Tao $[18,19]$ studied the effect of the location of the VG along the channel and its dimensions and shape influences on the heat transfer enhancement and pressure loss in a rectangular channel for Reynolds number of 1600. Based on their results, they concluded that increasing the VG frontal area results in increasing both heat transfer and pressure loss. Also, by making some changes in the VG dimensions, like increasing its span and decreasing its height, in a way that its frontal area is kept constant, it leads to achieve higher heat transfer enhancement while maintaining, or even decreasing, the pressure loss in the channel. Due to the presence of the VG, the cross-sectional flow area of the channel decreases, and the fluid velocity around the VG increases for the same flow rate. As a result, the strength of the generated LV increases, leading to an enhancement in the heat transfer. On the other hand, this decrease in the crosssectional flow area leads to a pressure drop increase.

Tiggelbeck et al. [20] investigated experimentally the heat transfer enhancement and pressure losses for different VG configurations (DW, RW, DWP and RWP) in developing turbulent flow.

In the open literature, most of the studies focus on the effect of the VG aspect ratio, its angle of attack for high Reynolds numbers [2000-8000], comparison of different geometries of VG and studying the heat transfer enhancement and the pressure losses.

In the present study, numerical investigation is performed on the effect of the angle of attack of a rectangular wing vortex generator (RWVG) on the thermal performance in laminar viscous flow (low Reynolds number) between two parallel plates. The generation of the induced vortices in low Reynolds laminar flows is more important than for that of turbulent flow. Different values of angle of attack $\alpha$ in the range $\left[0^{\circ}-30^{\circ}\right]$ are considered for a constant Reynolds number. Local and global Nusselt number and friction coefficients distributions within the channel are assessed. Also, the position and strength of the vortices being created in the channel are studied, highlighting their effect on the heat transfer performance.

\section{Problem description}

\subsection{Governing equations}

The flow field is governed by three-dimensional (3D) steadystate continuity and momentum equations. The fluid is considered to be incompressible Newtonian with constant thermophysical properties.

Continuity equation:

$\nabla \cdot \vec{u}=0$

Momentum equation:

$\rho(\vec{u} \cdot \nabla) \vec{u}=-\nabla p+\mu \nabla^{2} \vec{u}$

Energy equation:

$\rho C_{p} \nabla \cdot(\vec{u} T)=k_{f} \nabla \cdot(\nabla T)$

where $\vec{u}$ is the velocity vector, $C_{p}$ is the specific heat at constant pressure, $p$ is the pressure, $\mu$ is the fluid dymanic viscosity, $T$ is the temperature, $\rho$ is the fluid density and $k_{f}$ its thermal conductivity.

\subsection{Numerical procedure}

The computational fluid dynamics software used to compute the Navier-Stokes and energy equations is Star-CCM+ [21], which is based on the finite volume method. The segregated flow solver is used, wherein the equations are computed in a segregated manner, i.e. one for each component of velocity, and one for pressure. The coupling between the momentum and continuity equations is achieved with a predictor-corrector approach. The convective terms in the governing equations for momentum and energy are discretized with the secondorder up-wind scheme and second-order central scheme for diffusion terms. The algebraic multi-grid (AMG) linear solver is used to solve the velocity, pressure and temperature with Gauss-Seidel relaxation scheme. 
The residual value $10^{-14}$ is considered as the convergence criterion for the solutions of the flow and the energy equations. Beyond this value, no significant changes are found in the values for velocity and temperature.

\subsection{Computational domain and boundary conditions}

The computational domain consists of one row of a rectangular wing (RW) placed in between two parallel plates as shown in Fig. 1 a. The computational domain has a height $H=20 \mathrm{~mm}$, a width $W=5 H$ and a length $L=15 H$. The RW is inserted near the entrance on the bottom wall at a distance $P=H$ away from the inlet as shown in Fig. 1 b. As stated in the introduction, the angle of attack $\alpha$ which is the angle between the VG reference line and the incoming flow in the $Z$ direction, is variable in a range from $0^{\circ}$ to $30^{\circ}$. Remaining dimensions are given in Table 1 in terms of the channel height $H$.

Taking into account that both lateral sides are considered to be symmetry relative to the plan $(x, z)$, the computational domain is considered to be the half of the VG transverse pitch, thus half of the main domain. Flow and heat transfer simulations are carried out for Reynolds number 456, with uniform inlet temperature set to $T_{i n}=293 \mathrm{~K}$. Wall surface temperature $T_{w}=333 \mathrm{~K}$ is set for both bottom and top channel walls, as well as for the VG walls.

\subsection{Mesh study}

A non-uniform polyhedral mesh is generated in the core of the computational domain. Near the channel walls and VG surfaces, a prism-layer refinement mesh is preferred due to the presence of high velocity, temperature and pressure gradients in these regions. An example of the mesh on a flow cross section is shown in Fig. 2.

To determine the appropriate mesh density, the solver is run several times with increasing mesh densities until no significant effect on the results is detected, then the solution is considered to be a grid independent solution. The mesh validity verification is performed by using the method proposed by Celik et al. [22] who used the grid convergence index $(G C I)$ and the apparent order of convergence $(c)$. In the present study the mesh refinement is assessed by means of the global Nusselt number. This dimensionless number represents the ratio of convective to conductive heat transfer defined as:

$N u=\frac{h D_{h}}{k_{f}}$

where $h$ is the heat transfer coefficient, $D_{h}$ is the hydraulic diameter equal to $2 \mathrm{H}$ and $k_{f}$ is the thermal conductivity of the working fluid (air).
Fig. 1 a Isometric view of the computational domain, $\mathbf{b}$ side view showing the attack angle $\alpha$

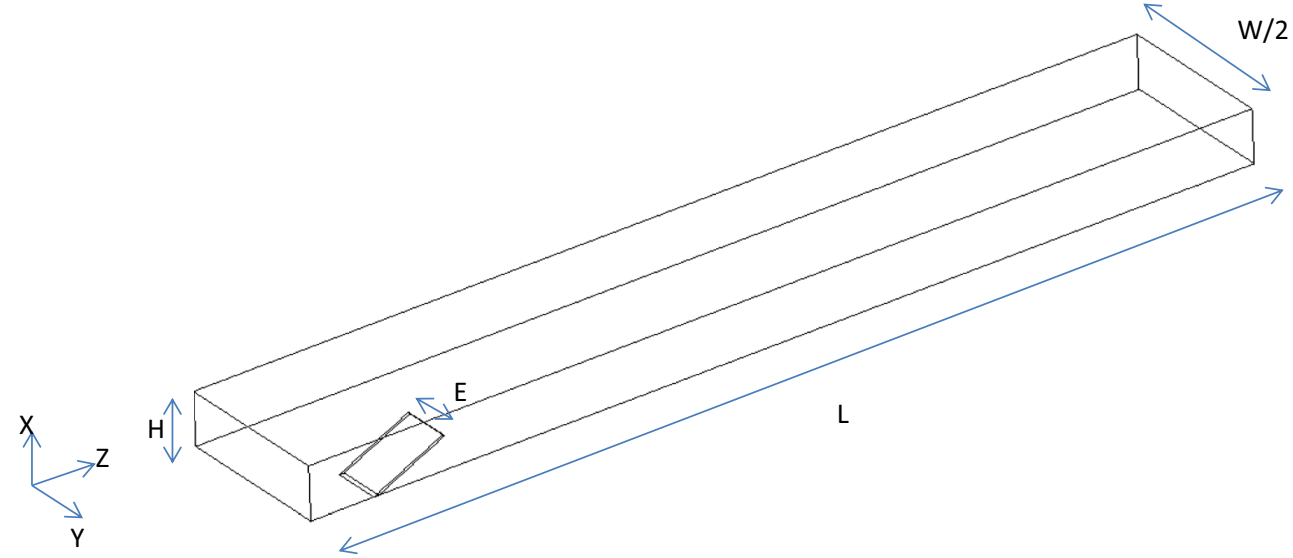

(a)

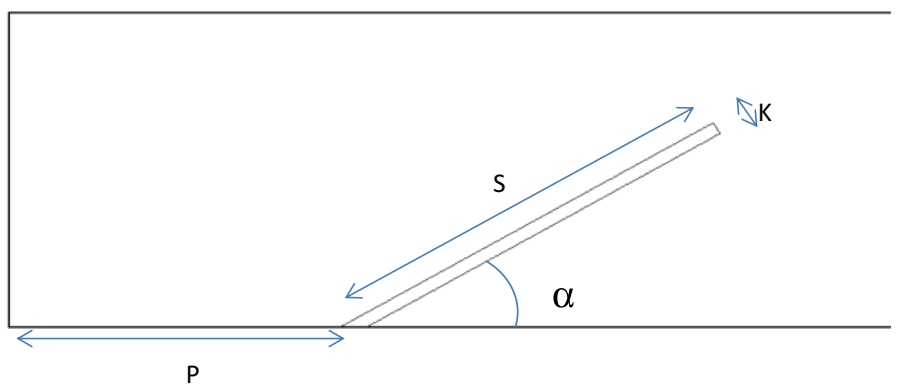

(b) 
Table 1 VG geometrical characteristics and computational domain dimensions

\begin{tabular}{lll}
\hline Channel height & $\mathrm{H}$ & $20 \mathrm{~mm}$ \\
Channel width & $\mathrm{W}$ & $5 \mathrm{H}$ \\
Channel length & $\mathrm{L}$ & $15 \mathrm{H}$ \\
Angle of attack & $\alpha$ & $0^{\circ}-30^{\circ}$ \\
VG length & $\mathrm{S}$ & $1.3 \mathrm{H}$ \\
VG width & $\mathrm{E}$ & $0.75 \mathrm{H}$ \\
Distance from the inlet to VG & $\mathrm{P}$ & $\mathrm{H}$ \\
VG thickness & $\mathrm{K}$ & $0.04 \mathrm{H}$ \\
\hline
\end{tabular}

In this equation, the global heat transfer coefficient is obtained from the logarithmic mean temperature difference:

$h=\frac{q}{A \Delta T_{l m}}$

where $q$ is the global rate of heat transfer defined in eq. (6), $A$ is the total heat transfer surface area and $\Delta T_{l m}$ is the logarithmic mean temperature difference defined in eq. (7),

$\dot{q}=\dot{m} C_{p} \Delta T$

where $\dot{m}$ is the mass flow rate, $C_{p}$ is the specific heat and $\Delta T$ is the temperature difference between the flow inlet and outlet $\left(\Delta T=T_{\text {out }}-T_{\text {in }}\right)$,

$\Delta T_{l m}=\frac{\left(T_{\text {in }}-T_{w}\right)-\left(T_{\text {out }}-T_{w}\right)}{\ln \left(\frac{T_{\text {out }}-T_{w}}{T_{\text {in }}-T_{w}}\right)}$

where $T_{\text {in }}$ is the inlet bulk temperature, $T_{\text {out }}$ is the outlet bulk temperature and $T_{w}$ is the wall temperature.

Three different mesh densities and their features are recapitulated in Table 2. The average mesh size is obtained by studying volume of each cell with respect to the total number

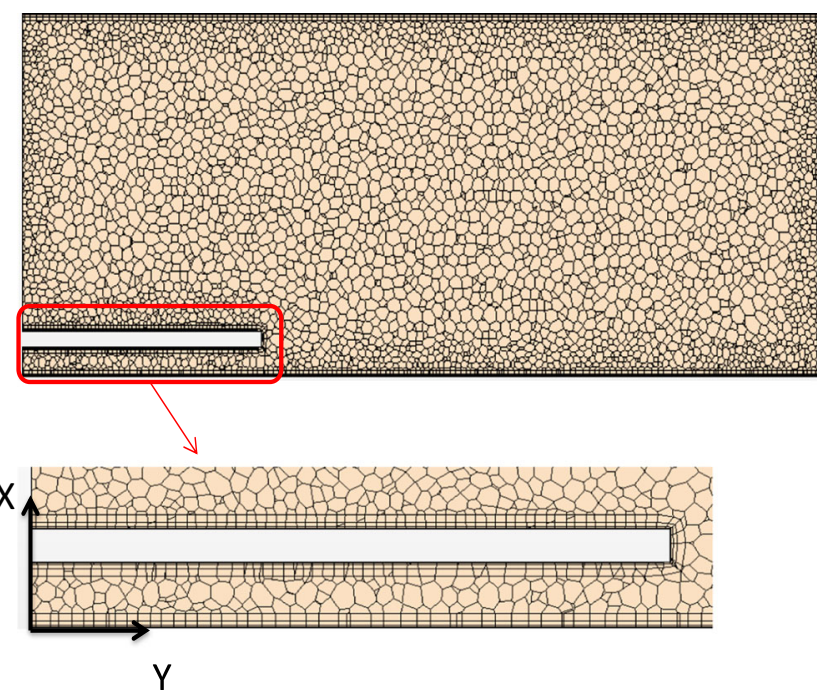

Fig. 2 Example of the mesh on a cross section showing the refinements around the VG and near the walls for $\alpha=10^{\circ}$ at $Z=1.7 \mathrm{H}$ of cells. It is desirable that the grid refinement factor value be greater than 1.3 as proposed by Celik et al. [22]. Based on eqs. (4) to (7), the Nusselt number is calculated for each mesh density then the convergence order $c$ and $G C I$ are computed.

Hence, it is found that the uncertainty in the fine-grid solution is $G C I=1.49 \%$ and the convergence order is $c=3.49$, which are both accepted values for which the results are considered to be grid independent. Based on the obtained values M2 is selected. For more details about the calculation of $c$ and GCI the reader can refer to Celik et al. [22].

Additionally local mesh study is also performed by considering transversal probe lines located at a distance $H$ downstream the wing (see Fig. 3) on both top and bottom channel walls, where wall heat fluxes transversal profiles can be analyzed. A third probe line is created in the core flow, at the center of the channel $(H / 2)$ and at the same distance downstream from the wing, to enable the velocity and temperature transversal profiles analysis for each mesh density considered.

The local relative errors between mesh densities M1 and M2 are calculated for various variables using the following equations:

- for heat flux $(q)$ at the walls:

$\varepsilon_{q}=\left|\frac{q_{M 1}-q_{M 2}}{q_{M 2}}\right|$

- for velocity $(v)$ in the core flow:

$\varepsilon_{v}=\left|\frac{v_{M 1}-v_{M 2}}{v_{M 2}}\right|$

- for dimensionless temperature $\Theta$ in the core flow, where $T$ is the temperature:

$\Theta=\frac{T-T_{w}}{T_{i n}-T_{w}}$

$\varepsilon_{\Theta}=\left|\frac{\Theta_{M 1}-\Theta_{M 2}}{\Theta_{M 2}}\right|$

Table 2 Mesh characteristics

\begin{tabular}{llcc}
\hline Mesh & M0 & M1 & \multicolumn{2}{l}{ M2 } \\
\hline Number of cells & 703,645 & $1,532,441$ & $3,289,799$ \\
Mesh size (mm) & 0.354 & 0.273 & 0.212 \\
Refinement factor & - & 1.3 & 1.3 \\
\hline
\end{tabular}




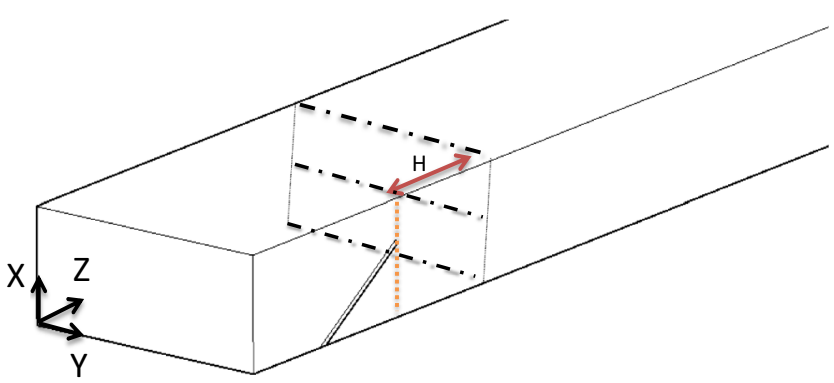

Fig. 3 Isometric view of the computational domain showing the location of the probe lines created downstream the VG for local mesh analysis

Based on the results presented in Fig. 4, it can be seen that the relative errors between $\mathrm{M} 1$ and $\mathrm{M} 2$ mesh densities always do not exceed $2.5 \%$ insuring good accuracy of the selected mesh density. Further validation is done hereunder by comparing the present computational results to a formerly published correlation.

\section{Results and discussions}

The major purpose of the present study is to analyze the effect of the angle of attack on the heat transfer and aeraulic performances, and then the flow topology and the temperature distribution are studied along the channel. Also investigations are done on the local and global parameters to check the effect of the angle of attack on the thermal performance enhancement.

\subsection{Empty channel validation}

Since the velocity and the temperature are set to be uniformed at the inlet of the computational domain, it is considered that the flow is a developing flow.

For thermally and hydraulically developing laminar air flow, the present numerical results are validated for local Nusselt number using Stephan correlation [23]. This

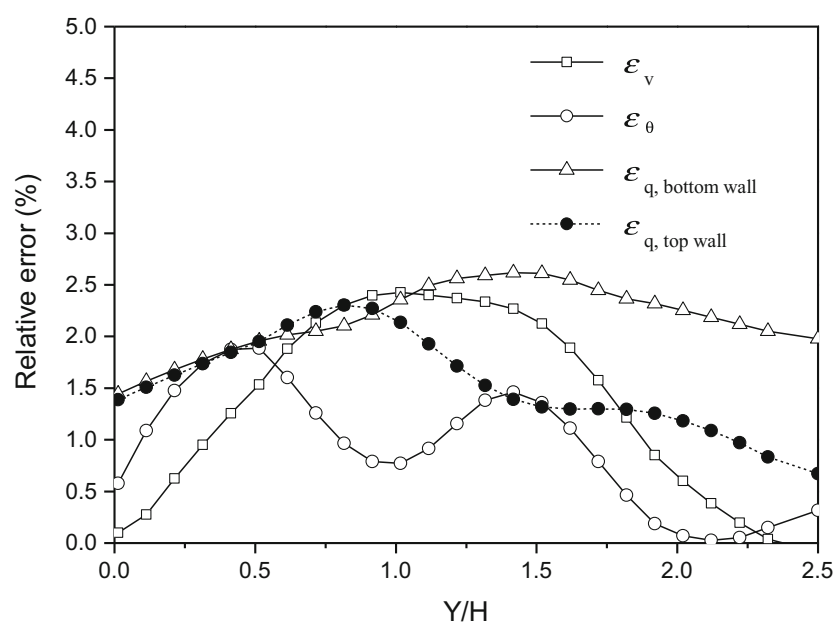

Fig. 4 Relative local error between the meshes M1 and M2 for bottom and top wall heat fluxes, velocity and dimensionless temperature in the core flow (at mid-channel height) correlation is valid in the range $0.1 \leq \operatorname{Pr} \leq 1000$ for parallel plate channels. The local Nusselt number is represented as followed:

$N u_{x}=7.55+\frac{0.024 x_{*}^{-1.14}\left(0.0179 \operatorname{Pr}^{0.17} x_{*}^{-0.64}-0.14\right)}{\left(1+0.0358 \operatorname{Pr}^{0.17} x_{*}^{-0.64}\right)^{2}}$
$x_{*}=\frac{x}{D_{h} \operatorname{RePr}}$

Where $\mathrm{x}$ represents the location along the channel starting from the inlet till the outlet of it.

Based on eqs. (12) and (13) the local Nusselt number is calculated and compared with computational results obtained for the empty channel. Figure 5 represents the results, where the average percentage error is $1.87 \%$ along the duct.

\subsection{Flow structure and temperature distributions}

In order to understand the flow behavior, its structure is first studied for two values of the angle of attack: $\alpha=10^{\circ}$ and $\alpha=$ $30^{\circ}$, for same Reynolds number $R e=456$.

Figure 6 represents the helicity distribution, where helicity is the product of the velocity with value of the vorticity, and the vorticity streamlines along the channel for different cross sections located downstream the VG, for $\alpha=10^{\circ}$ on Fig. 6 a and for $\alpha=30^{\circ}$ on Fig. 6 b. By studying these two parameters, the generation of the vortices due to the VG is highlighted. Also the intensity of the vortex and its location along the channel is studied by taking different planes. $Z=2.5 H$ represents the plane just after the VG, where the vortices are generated due to the presence of high velocity gradients. As shown in Fig. 6, rotating vortices are generated downstream from the VG. At $Z=2.5 H$ there is a negative value of helicity, this is due to the reverse vortices generated by the VG and their effect is detected till the end of the channel.

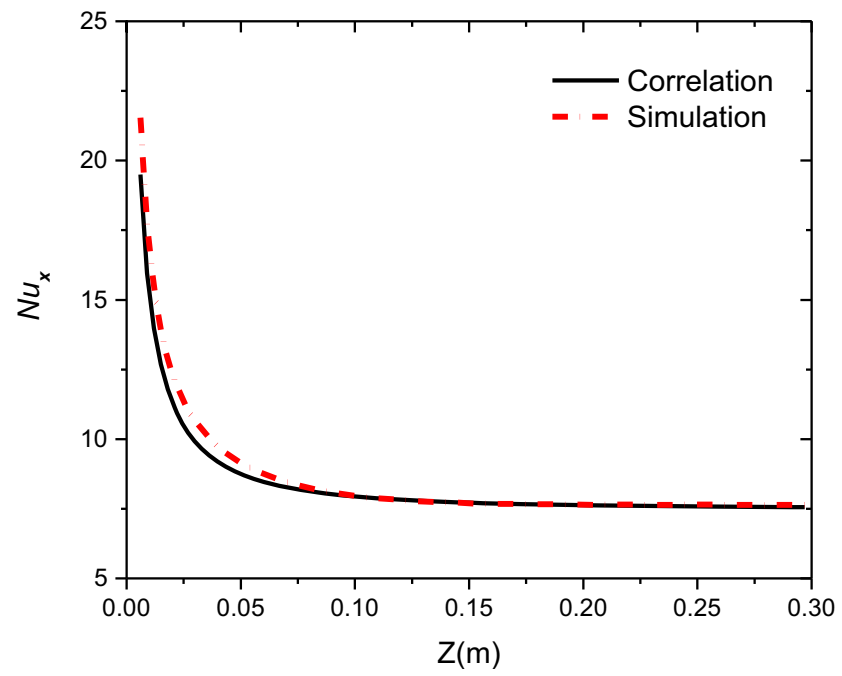

Fig. 5 Validation for empty channel simulation 
By comparing $\alpha=10^{\circ}$ and $\alpha=30^{\circ}$, it is clear how the angle of attack $\alpha$ effects the generation of the vortices, and their effect on the flow topology along the channel. For $\alpha=$ $30^{\circ}$, the generated vortices are more intense than that of $\alpha=$ $10^{\circ}$, also at $Z=5 H$ an induced secondary vortex is detected for the case of $\alpha=30^{\circ}$.

As the angle of attack $\alpha$ increases, it is clear that the effect of the vortices generated last longer along the channel reaching until to a location of $Z=15 H$ which represents almost the outlet of the channel. For the case of $\alpha=10^{\circ}$ at $Z=$ $15 H$, the effect of the VG starts to fade away, which is supported by both the streamlines and the helicity contours. On the other hand, for the case of $\alpha=30^{\circ}$ at $Z=15 \mathrm{H}$, the effect of the generated vortices is dominant till the end of the channel.

In Fig. 7 the temperature distribution is represented for different flow cross sections at locations $Z / H$ in the range [2.5-15] and for $\alpha=10^{\circ}$ and $30^{\circ}$. Figure 7 illustrates that the streamwise vortices previously mentioned affect the temperature distribution downstream the VG, especially for the highest angle. Indeed in the common flow down region, between the two main vortices (i.e. in the symmetry plane of the RW), hot fluid particles are ejected from the top wall towards the flow core due to upwash effect, while the thickness of the thermal boundary layer on the bottom wall is found to decrease due to downwash effect. In the common flow up region (i.e. between two neighboring vortex pairs) the upwash effect ejects near-wall hot fluid from the bottom wall towards the flow core. This mixing process and thermal boundary thinning are clearly seen for the highest attack angle value, due to the fact that the vortices are more energetic, and cover larger area in the flow cross section. Then, the heat transfer enhancement seems to be dependent of the wing attack angle value. This is assessed quantitatively and discussed in the next sections by representing the streamwise development of the Nusselt number and the vorticity strength.

The streamwise variation of the area-weighted span-averaged helicity is represented in Fig. 8. Where the dashed vertical lines represent the head $(\mathrm{Z}=1.2 \mathrm{H})$ and the tail $(2 \mathrm{H}<\mathrm{Z}<$ $2.4 \mathrm{H})$ of the VG.

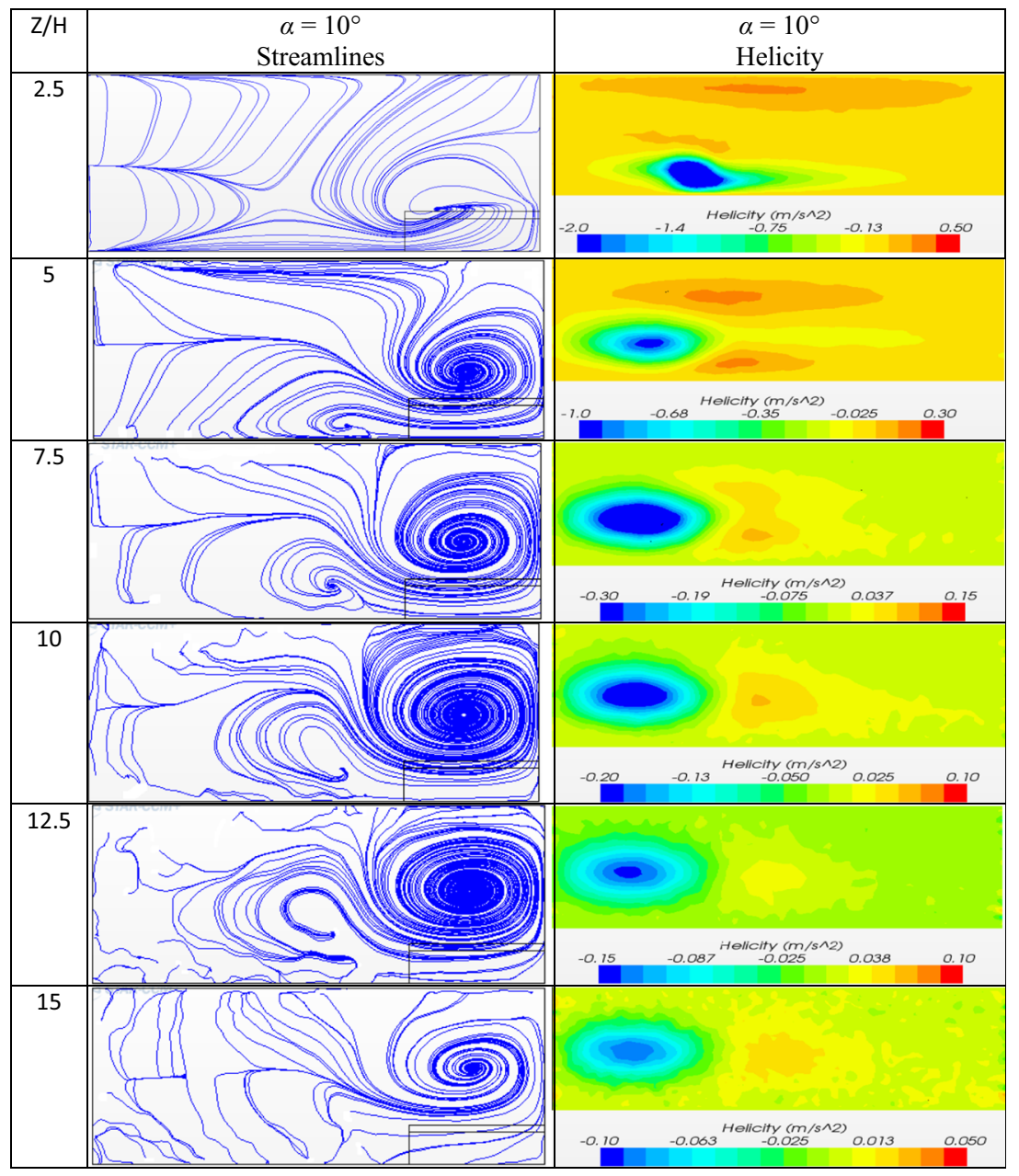

(a)

Fig. 6 Helicity distribution (right side) and streamlines (left side) for $R e=456$ at locations $Z / H$ in the range [2.5-15], for (a) $\alpha=10^{\circ}$, (b) $\alpha=30^{\circ}$ 


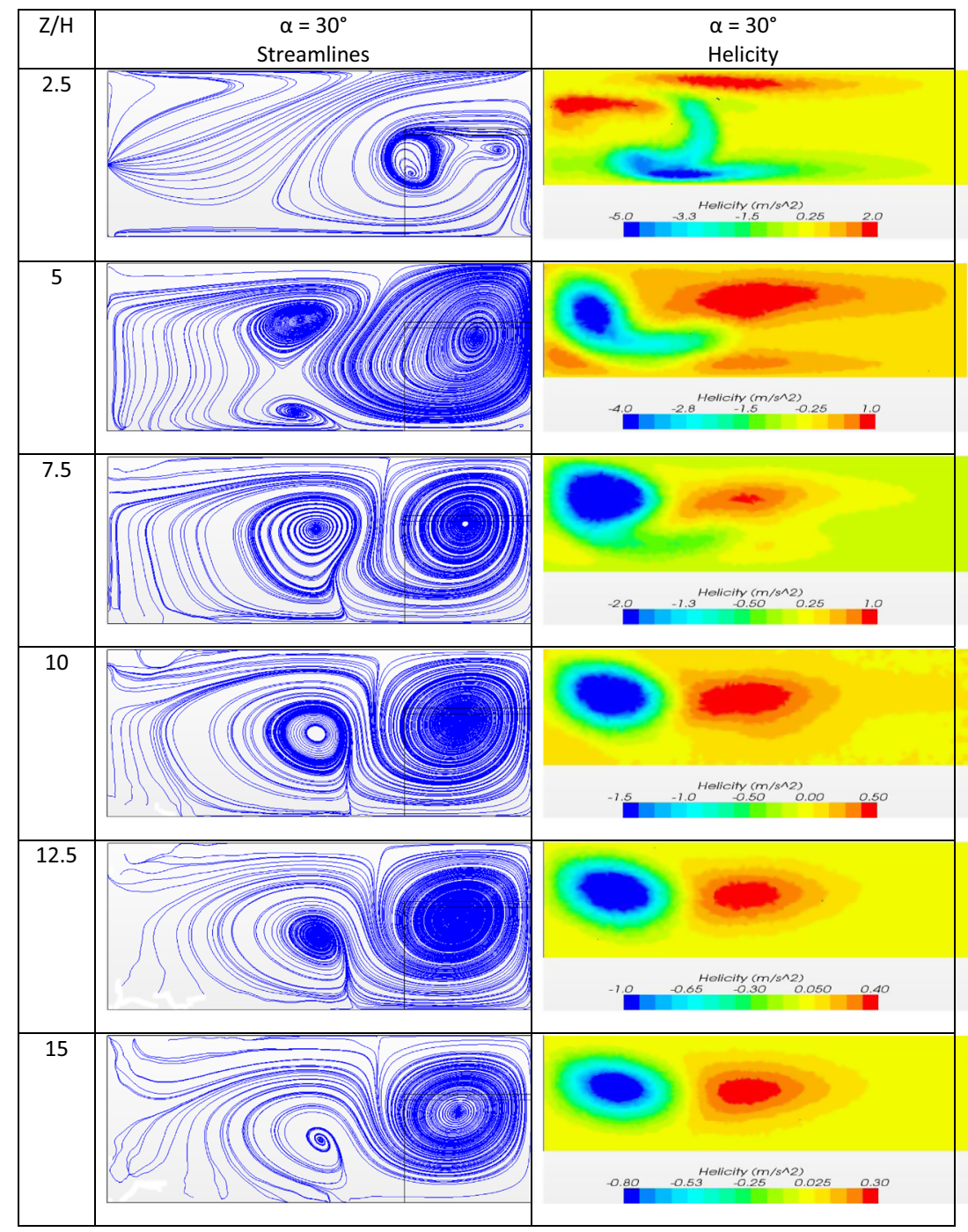

(b)

Fig. 6 continued.

Fig. 7 Temperature distribution for $R e=456$ on different flow cross sections at locations $Z / H$ in the range [2.5-15], for $\alpha=10^{\circ}$ (left side) and $\alpha=30^{\circ}$ (right side)

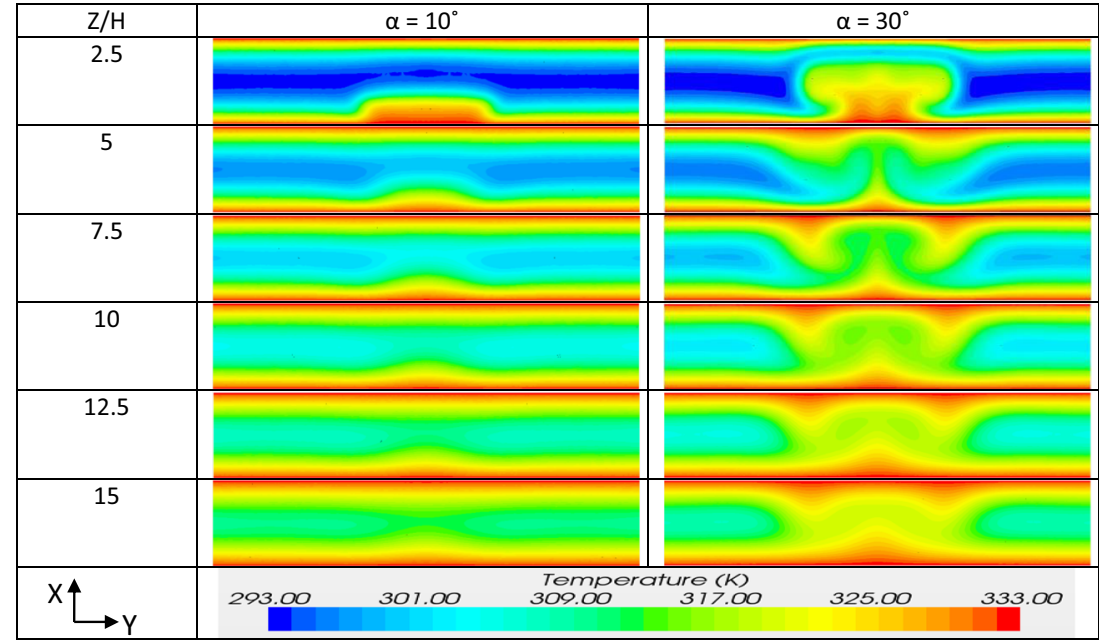




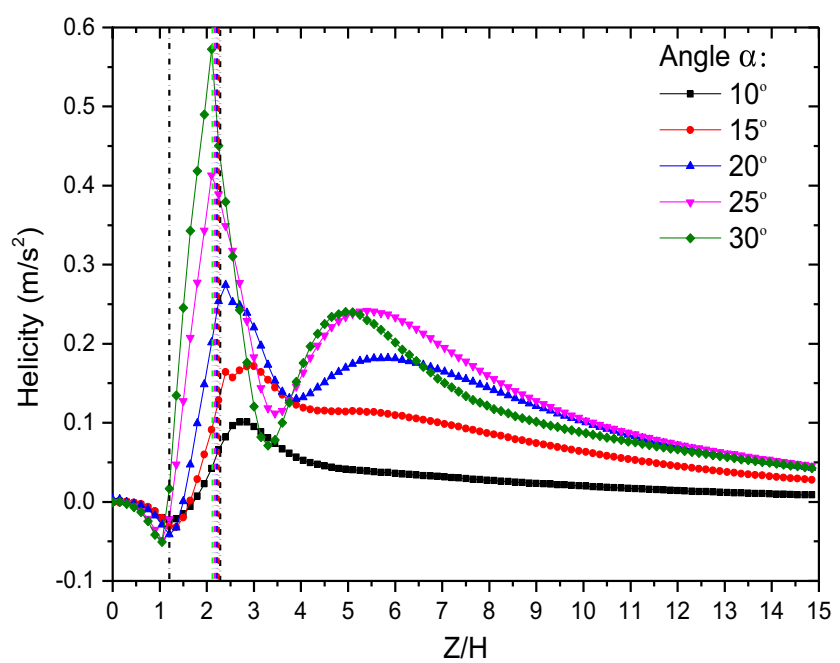

Fig. 8 Variation of span-averaged helicity along the parallel plate for $R e=456$

It is observed that the helicity values start to decrease at the entrance of the channel reaching even negative values at a position just upstream the VG. The negative values are obtained due to the direction of the rotation of the main vortex. Downstream the flow encounters the VG, the averaged helicity is found to increase and reach its maximum value in the very near vicinity of the VG trailing edge because of the generated vortices, for location $\mathrm{Z}$ between $2 H$ and $3 H$. It can be noticed that the helicity peak for the case $\alpha=30^{\circ}$ is about 6 times higher than that for $\alpha=10^{\circ}$ due to higher energetic vortices.

For $\alpha=10^{\circ}$ and $\alpha=15^{\circ}$ the profile of the streamwise evolution of the vorticity is similar having a maximum at the VG tail and decreasing continuously along the channel because of the dissipation of the longitudinal vortices. Whereas for higher $\alpha$ values $\left(20^{\circ}, 25^{\circ}\right.$ and $\left.30^{\circ}\right)$, secondary peaks are generated downstream from the first one due to the induced vortices generated. It is interesting to note that the higher the angle of attack, the higher the primary generated peak and also the higher the secondary peaks. However, at a distance from the secondary peak, for the case $\alpha=20^{\circ}$ the helicity sustains a higher value, from $Z=6.5 \mathrm{H}$ compared to $\alpha=30^{\circ}$. For the case $\alpha=25^{\circ}$, the value of the secondary generated peak is almost the same as that of the case $\alpha=30^{\circ}$ and the helicity continuous to exhibit higher values along the channel compared to the other cases.

In Fig. 9 the streamwise variation of the dimensionless location of the main vortex is presented; it corresponds to the distance between the vortex core and the bottom wall. The position of the core of the main vortex starts in an increasing profile for all the values of attack angle just after the VG tail at $\mathrm{Z}=2.5 \mathrm{H}$. It is observed that by increasing the value of attack angle $\alpha$, the position of the main vortex along the channel increases rapidly. For the cases $\alpha<25^{\circ}$, the position of the main vortex core increases gradually along the channel, until

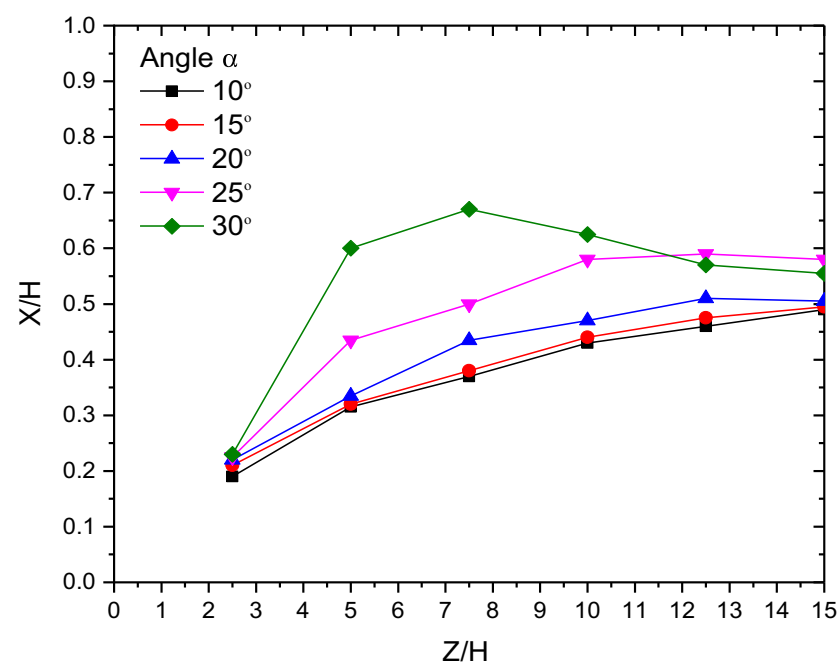

Fig. 9 Dimensionless location of the main vortex along the channel for $\operatorname{Re}=456$

reaching values for $X$ around $0.50 H$. It is interesting to note that for $\alpha=30^{\circ}$ the location of the core of the main vortex reaches a maximum value $X=0.65 H$ at the location $Z=7.5 H$ and then decreases to $X=0.55 H$ at the channel output.

As the dimensionless location of the core of the main vortex reaches around the half of the channel height, it leads to a better mixing of the fluid in the channel. It mixes the hot particles located in the vicinity of both lower and upper boundaries with that of the central ones having a lower temperature values. As a result, the heat transfer rate increases, due to the high temperature gradient and then the Nusselt number. The variation in the Nusselt number is then studied in the next sections, in order to improve the comprehension of the effect of the angle of attack $\alpha$ on the heat transfer enhancement.

\subsection{Local performances}

The streamwise distribution of the span-averaged Nusselt number at the bottom wall is plotted on Fig. 10 a for values of attack angles $\alpha$ in the range $\left[10^{\circ}-30^{\circ}\right]$ as well as for the case of the empty channel, simulated with the same operating conditions except the VG which is removed. At the inlet of the channel, the values of $N u$ starts to decrease until the location $Z=1.2 H$ which represents the beginning location of the VG. From this location, the Nusselt number value starts to increase due to the heat transfer enhancement as a result of the generated vortices until it reaches the VG tail. Further downstream, for all the simulated cases, the Nusselt value drops to a minimum value, at a location of $Z=1.2 \mathrm{H}$ which represents the beginning location of the $\mathrm{VG}$, and this minimum value is found to be even below the empty channel case, for the cases $\alpha$ in the range $\left[10^{\circ}-20^{\circ}\right]$. For low values of $\alpha\left[10^{\circ}-20^{\circ}\right]$, the area under the VG is greater than that of the higher angles. As a result of this, a dead zone is created with zero velocity 
leading to a drop in the convective heat transfer thus drop in the Nusselt number. Downstream the VG, the Nusselt number increases until attains a second peak at locations $\mathrm{Z}$ in the range $[3.5 \mathrm{H}-5 \mathrm{H}]$. For all the values of $\alpha$, a similar profile is obtained where after the second peak the Nusselt number decreases along the longitudinal direction of the channel. However, the higher the angle of attack, the higher the Nusselt number.

Figure $10 \mathrm{~b}$ represents the streamwise distribution of the span-averaged Nusselt number at the top wall, plotted for values of attack angles $\alpha$ in the range $\left[10^{\circ}-30^{\circ}\right]$ and for the case of the empty channel. The Nusselt number for the top wall starts to decreases in the inlet of the channel and at the location $Z=1.2 H$, the value starts to increase reaching its maximum at $Z=2.3 H$ for all values of $\alpha$ except that of $\alpha=$ $10^{\circ}$ which continues to decrease smoothly along the longitudinal direction of the channel. The higher the angle of attack, the higher the Nusselt number peak. The drop in the Nusselt curve below the value of the empty channel also occurs on the top wall for the cases of $\alpha=20^{\circ}, 25^{\circ}$ and $30^{\circ}$. It occurs for $\alpha=$ $20^{\circ}$ at $Z=9 \mathrm{H}, \alpha=25^{\circ}$ at $Z=6 \mathrm{H}$ and $\alpha=30^{\circ}$ at $Z=5 \mathrm{H}$. This behavior will be explained in the next section.

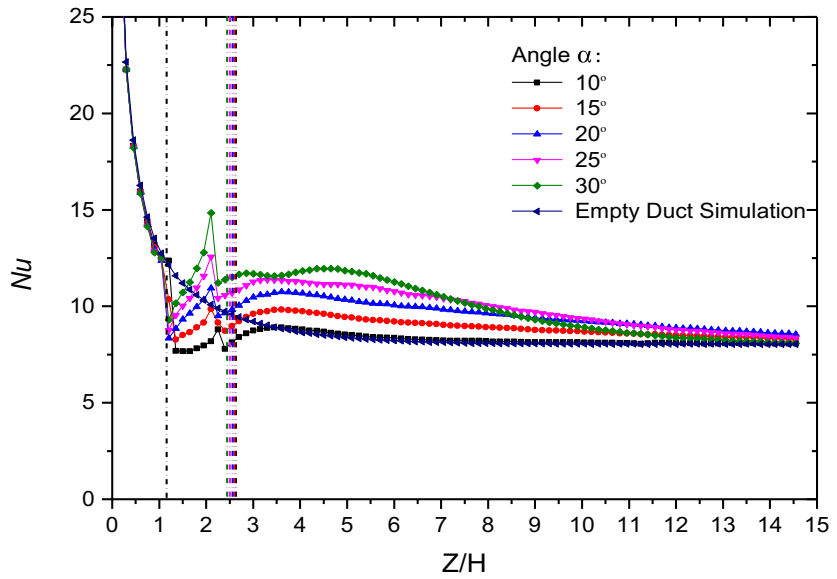

(a)

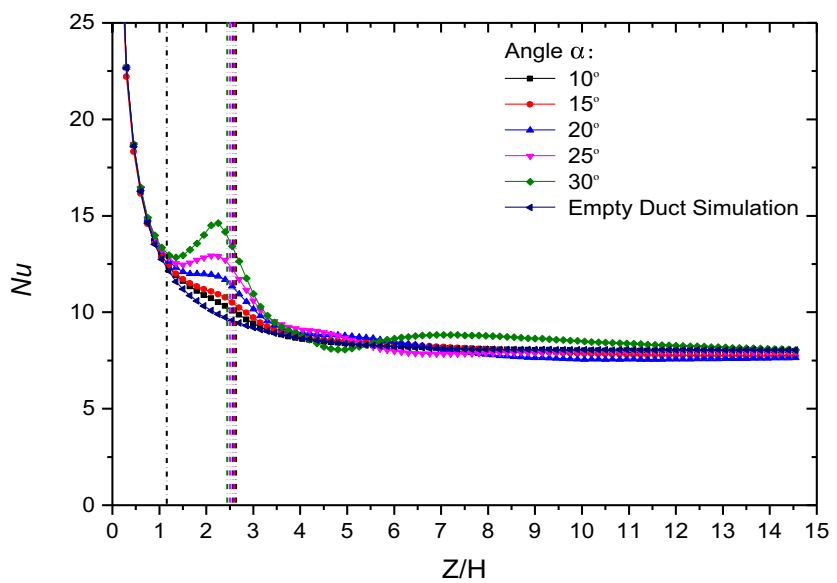

(b)
To understand the drop of the Nusselt number in some cases of channel equipped with VG below the results of empty channel, a study is done to check the near-wall velocity distributions. In Fig. 11, the near wall velocity is studied for the values of $\alpha$ in the range $\left[10^{\circ}-30^{\circ}\right]$ at two locations: $X=H / 10$, i.e. a plane parallel to the bottom plan located at a small distance $(H / 10)$, knowing that $X=0$ represents the bottom wall. Another plane of investigation is defined at $\mathrm{X}=9 \mathrm{H} / 10$ away from the bottom wall. The velocity value for the case $\mathrm{X}=\mathrm{H} /$ 10 at a position under the VG highlighted by a black circle is zero: a dead zone is being created under the VG where due to zero velocity there will be negligible convective heat transfer. As a result the Nusselt number at that location is dropping even below the empty channel. On the other hand, for the case $X=9 \mathrm{H} / 10$, for $\alpha=20^{\circ}, 25^{\circ}$ and $30^{\circ}$, very low values of velocity is detected on the near top wall too. As a result, for $\alpha=$ $20^{\circ}, 25^{\circ}$ and $30^{\circ}$ it appears very weak convective heat transfer on the top wall at the positions highlighted by a circle. This is supported by the values of the Nusselt number that are represented in Fig. 10 for the top wall case.

To evaluate the pressure losses induced by the VG, Fanning friction factor $f$ is calculated from the pressure gradient obtained from the simulation. Darcy's friction can be represented by:

$f_{\text {darcy }}=2 \frac{D_{h}}{L} \cdot \frac{\Delta p}{\rho U^{2}}=\frac{4 H \Delta p}{L \rho U^{2}}$

thus

$f=\frac{f_{\text {darcy }}}{4}=\frac{H \Delta p}{L \rho U^{2}}$

where $H$ is the channel height, $\Delta p$ is the pressure drop between the inlet and the outlet, $L$ is the length of the channel, $\rho$ is the fluid density and $U$ is the mean flow velocity.

In order to calculate the local Fanning friction factor, the following relation is used:

$f(z)=\frac{f(z)_{\text {darcy }}}{4}=\frac{H \Delta p(z)}{Z \rho U^{2}}$

where $\Delta p(z)$ is the pressure drop between the inlet and a cross section at location $Z$.

In Fig. 12, the streamwise variation of the friction factor is represented. The profile of the curve is similar in all the case. It starts to drop along the length of the channel until it reaches the VG leading edge. At that point, a gradual increase starts to appear reaching its maximum value at the VG tail of the VG, downstream which the curve continues to drop making an asymptote with the empty channel curve. From the leading edge of the VG, the higher the angle of attack, the higher the friction factor.

Fig. 10 Nusselt number for $R e=456$ at (a) bottom wall, (b) top wall 
Fig. 11 Velocity magnitude contours at location $X=H / 10$ away from bottom wall (left side) and top wall (right side) for $R e=$ 456

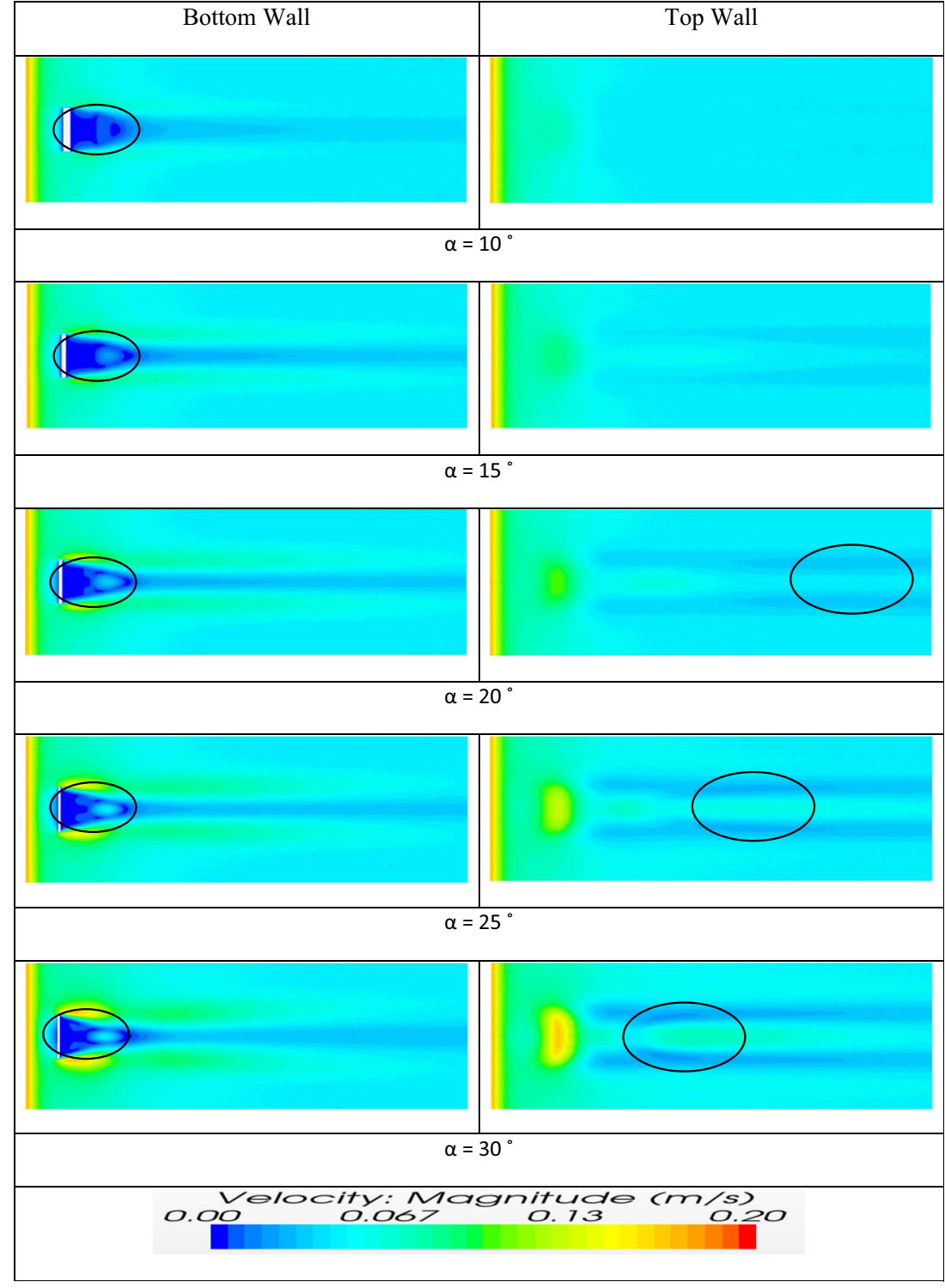

\subsection{Global performances}

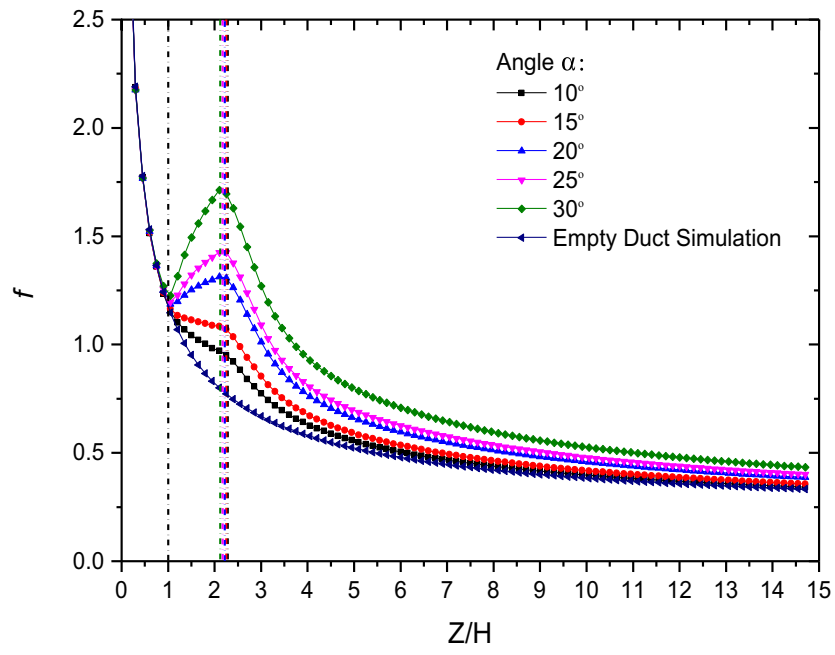

Fig. 12 Friction factor streamwise variation for $R e=456$
In order to study the global effect of the angle of attack $\alpha$ of the VG on the enhancement of the heat transfer, global values of the Nusselt number, the friction factor and the thermal enhancement factor are presented and discussed in this section.

Figure 13 represents the global Nusselt number plotted versus angle of attack $\alpha$. The Nusselt number is calculated using eq. (4). The Nusselt number is found to monotonically increase with the value of the angle of attack. By comparing the empty channel and $\alpha=10^{\circ}$, there is an increase of about $1 \%$ in the Nusselt number on the other hand for $\alpha=30^{\circ}$ this increase reaches to a value of $10 \%$.

The global friction factor is plotted versus the angle of attack in Fig. 14. The friction factor is calculated using eq. (15). As the angle of attack $\alpha$ increases, the friction factor increases too. For higher values of angle $\alpha$ the pressure drop 


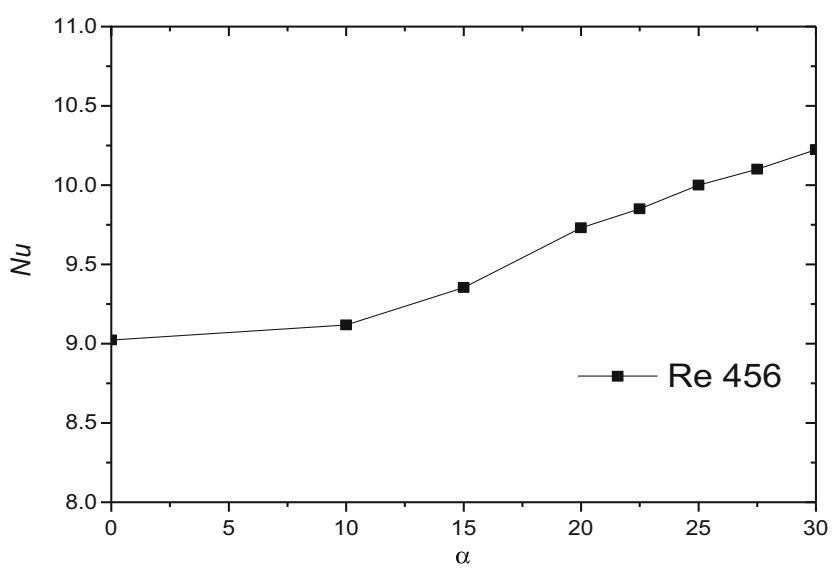

Fig. 13 Global Nusselt number for $R e=456$

is increased, as a result the pressure gradient is increased leading to higher values of friction factor. This increase reaches to a value of $30 \%$ by comparing the empty channel with respect to that of $\alpha=30^{\circ}$.

Based on the previously mentioned relations of the Nusselt number and the friction factor, a thermal enhancement factor is calculated using the following relation:

$\eta=\left(\frac{N u}{N u_{0}}\right)\left(\frac{f}{f_{0}}\right)^{(-1 / 3)}$

Where $N u_{0}$ and $f_{0}$ are the values of respectively Nusselt number and friction factor for the empty channel.

Figure 15 represents the thermal enhancement factor versus the angle of attack. The value of the thermal enhancement factor starts to increase with the increase of the angle of attack $\alpha$. For values of $\alpha=10^{\circ}$ and $\alpha=15^{\circ}$, there is a linear increase reaching to a value of 1.012representing $1.2 \%$ of enhancement. For $\alpha=20^{\circ}$, there is an increase in the thermal enhancement factor reaching its maximum value of 1.043 representing $4.3 \%$ of enhancement for the case $\alpha=25^{\circ}$, beyond which the enhancement factor slightly drops. This increase in the

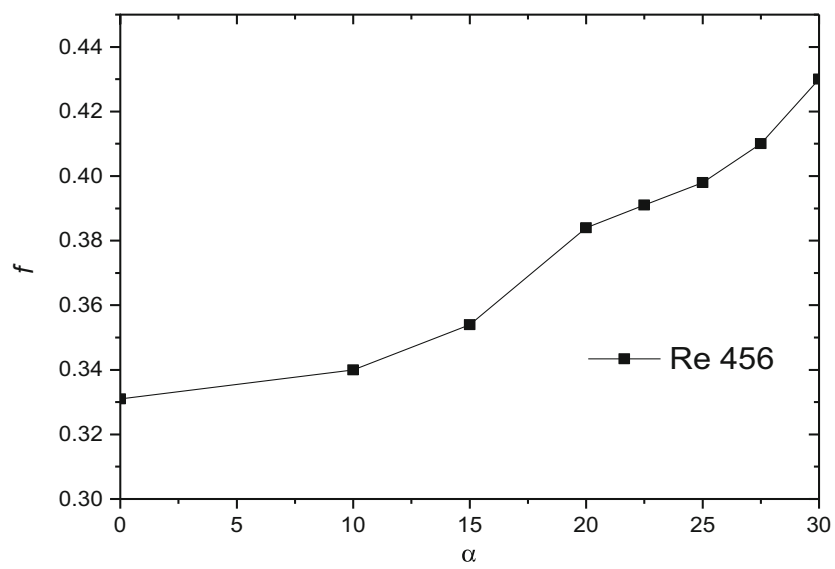

Fig. 14 Global friction factor for $R e=456$

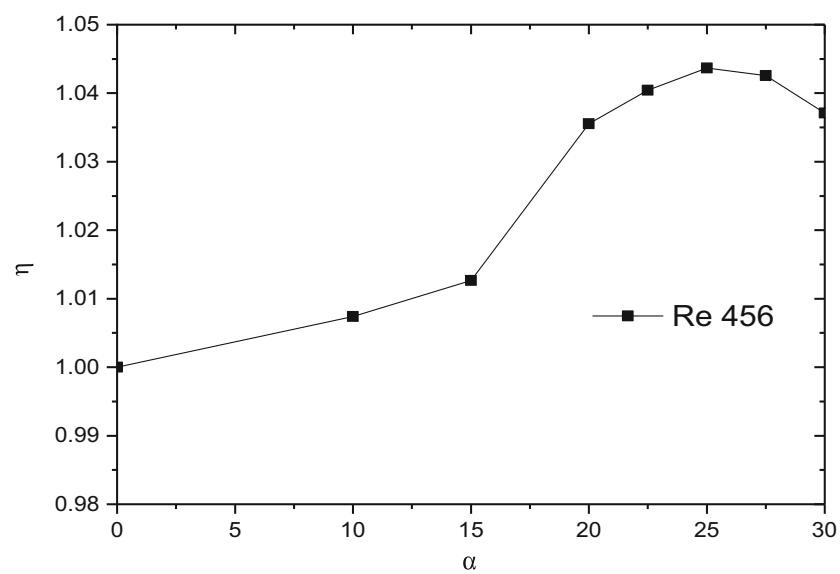

Fig. 15 Thermal enhancement factor for $R e=456$

enhancement is supported by the helicity curve represented in Fig. 6, where secondary peaks are generated for $20^{\circ} \leq \alpha \leq 30^{\circ}$.

Thus it can be considered that for the operating condition $R e=456$, the optimum angle of attack providing the best thermal enhancement is $25^{\circ}$ and not the highest angle, this is supported by the helicity study which have shown the detected secondary vortices where the curve of $\alpha=25^{\circ}$ sustains higher values along the channel compared to the other tested angles.

\section{Conclusion}

In this study, the heat transfer and fluid flow behavior in a parallel plate channel with rectangular wing vortex generator (RWVG) inserted on the bottom wall is numerically investigated. For different values of angle of attack $\alpha$ both the local and the global parameters are calculated and studied to figure out the effect of the angle of attack on the heat transfer enhancement. The main outcomes of this study can be summarized as followed.

First, for high values of angle of attack (close to $\alpha=30^{\circ}$ ), the generated vortices get wider and more energetic along the longitudinal direction compared with low values (i.e. $\alpha=10^{\circ}$ ).

Second, it is observed that the helicity increases just after the flow encounters the VG where the vortices are first formed. The helicity peak for $\alpha=30^{\circ}$ is about 6 folds higher than that for $\alpha=10^{\circ}$ meaning higher energetic vortices.

Third, it is observed that for $\alpha$ in the range $\left[10^{\circ}-25^{\circ}\right]$, the dimensionless vertical position $X / H$ of the main vortex starts at low position and increases along the channel until it reaches a maximum value at the outlet of the channel $(Z=15 H)$ around $X=0.5 H$ representing the middle of the channel, for the case $\alpha=$ $25^{\circ}$, it reaches a maximum value of $X=0.58 \mathrm{H}$. On the other hand, for the case $\alpha=30^{\circ}$, the dimensionless $X / H$ position reaches a maximum value of $X=0.68 H$ before the outlet channel and then drops back to attain $0.55 \mathrm{H}$ at the end of the channel. 
Finally, the thermal enhancement factor starts to increase with the angle of attack until it reaches a maximum value of 1.043 representing $4.3 \%$ of enhancement at $\alpha=25^{\circ}$, beyond which its value decreases. It can be considered that the optimum angle of attack of the vortex generator which leads to the best enhancement under these operating conditions is $25^{\circ}$ and not the highest angle.

\section{Compliance with ethical standards}

Conflict of interest On behalf of all authors, the corresponding author states that there is no conflict of interest.

Charbel Habchi (May 10, 2017).

\section{References}

1. Khoshvaght-Aliabadi M, Zangouei S, Hormozi F (2015) Performance of a plate-fin heat exchanger with vortex-generator channels: 3D-CFD simulation and experimental validation. Int $\mathrm{J}$ Therm Sci 88:180-192

2. Hachem F, Khaled M, Ramadan M, Habchi C (2014) Boundary Layer Development on a Concave Surface: Flow Visualization and Hot Wire Velocity Measurements. J Energy Power Eng 8: $1177-1182$

3. Alahyari Beig S, Mirzakhalili E, Kowsari F (2011) Investigation of optimal position of a vortex generator in a blocked channel for heat transfer enhancement of electronic chips. Int J Heat Mass Transf 54: $4317-4324$

4. Jiang Y, Zheng Q, Dong P, Zhang H, Yu F (2014) Research on heavy-duty gas turbine vane high efficiency cooling performance considering coolant phase transfer. Appl Therm Eng 73:1175-1191

5. Basu DN, Bhattacharyya S, Das PK (2014) A review of modern advances in analyses and applications of single-phase natural circulation loop in nuclear thermal hydraulics. Nucl Eng Des 280: 326-348

6. Fiebig M (1998) Vortices, Generators and Heat Transfer. Chem Eng Res Des 76:108-123

7. Habchi C, Russeil S, Bougeard D, Harion JL, Lemenand T, Della Valle D, Peerhossaini H (2012) Enhancing heat transfer in vortex generator-type multifunctional heat exchangers. Appl Therm Eng 38:14-25

8. Anxionnaz Z, Cabassud M, Gourdon C, Tochon P (2008) Heat exchanger/reactors (HEX reactors): Concepts, technologies: Stateof-the-art. Chem Eng Process Process Intensif 47:2029-2050
9. Ghanem A, Habchi C, Lemenand T, Della Valle D, Peerhossaini H (2013) Energy efficiency in process industry - High-efficiency vortex (HEV) multifunctional heat exchanger. Renew Energy 56:96104

10. Tiggelbeck S, Mitra NK, Fiebig M (1992) Flow Structure and Heat Transfer in a Channel with Multiple Longitudinal Vortex Generators. Exp Thermal Fluid Sci 5:425-436

11. Jacobi AM, Shah RK (1995) Heat Transfer Surface Enhancement through the Use of Longitudinal Vortices: A Review of Recent Progress. Exp Thermal Fluid Sci 11:295-309

12. Habchi C, Lemenand T, Della Valle D, Pacheco L, Le Corre O, Peerhossaini H (2011) Entropy production and field synergy principle in turbulent vortical flows. Int J Therm Sci 50:2365-2376

13. Mohand Kaci H, Habchi C, Lemenand T, Della Valle D, Peerhossaini H (2010) Flow structure and heat transfer induced by embedded vorticity. Int J Heat Mass Transf 53:3575-3584

14. Zhou G, Feng Z (2014) Experimental investigations of heat transfer enhancement by plane and curved winglet type vortex generators with punched holes. Int J Therm Sci 78:26-35

15. Wang CC, Chen KY, Lin YT (2015) Investigation of the semidimple vortex generator applicable to fin-and-tube heat exchangers. Appl Therm Eng 88:192-197

16. Fiebig M (1995) Embedded Vortices in Internal Flow: Heat Transfer and Pressure Loss Enhancement. Int J Heat Fluid Flow 16:376-388

17. Fiebig P, Kallweit N, Mitra S (1991) Tiggelbeck, Heat Transfer enhancment and drag by longitudinal vortex generators in channel flow. Exp Thermal Fluid Sci 4(1):103-114

18. Wu JM, Tao WQ (2008) Numerical study on laminar convection heat transfer in a channel with longitudinal vortex generator. Part B: Parametric study of major influence factors. Int J Heat Mass Transf 51:3683-3692

19. Wu JM, Tao WQ (2008) Numerical study on laminar convection heat transfer in a rectangular channel with longitudinal vortex generator. Part A: Verification of field synergy principle. Int J Heat Mass Transf 51:1179-1191

20. Tiggelbeck S, Mitra NK, Fiebig M (1994) Comparison of wingtype vortex generators for heat transfer enhancement in channel flows. J Heat Transf 116:880-885

21. CD-adapco, STAR CCM+, Version 8, Users Manual. http://www. cd-adapco.com/products/star-ccm/documentation

22. Celik IB, Ghia U, Roache PJ, Freitas CJ, Coleman H, Raad PE (2008) Procedure for Estimation and Reporting of Uncertainty Due to Discretization in CFD Applications. J Fluids Eng 130: 078001-078001

23. Bejan A, Kraus A (2003) Heat Transfer Handbook, vol 1. Wiley, New Jersey, pp 395-438 